\title{
Shadow images of Kerr-like wormholes
}

\author{
Muhammed Amir, ${ }^{1, *}$ Kimet Jusufi, ${ }^{2,3, \dagger}$ Ayan Banerjee, ${ }^{1, \ddagger}$ and Sudan Hansraj ${ }^{1, \S}$ \\ ${ }^{1}$ Astrophysics and Cosmology Research Unit, School of Mathematics, \\ Statistics and Computer Science, University of KwaZulu-Natal, \\ Private Bag X54001, Durban 4000, South Africa \\ ${ }^{2}$ Physics Department, State University of Tetovo, \\ Ilinden Street nn, 1200, Tetovo, North Macedonia \\ ${ }^{3}$ Institute of Physics, Faculty of Natural Sciences and Mathematics, \\ Ss. Cyril and Methodius University, \\ Arhimedova 3, 1000 Skopje, North Macedonia
}

(Dated: September 10, 2019)

\begin{abstract}
Investigations of shadows of astrophysical entities constitute a major source of insight into the evolution of compact objects. Such effects depend on the nature of the compact object and arise on account of the strong gravitational lensing that casts a shadow on the bright background. We consider the Kerr-like wormhole spacetime (Phys. Rev. D 97:024040, 2018), which is a modification of the Kerr black hole that degenerates into wormholes for nonzero values of the deviation parameter $\lambda^{2}$. The results suggest that the Kerr spacetime can reproduce far away from the throat of the wormhole. We obtain the shapes of the shadow for the Kerr-like wormholes and discuss the effect of the spin $a$, the inclination angle $\theta_{0}$, and the deviation parameter $\lambda^{2}$ on the size and nature of the shadow. As a consequence, it is discovered that the shadow is distorted due to the spin as well as the deviation parameter and the radius of the shadow decreases with $\lambda^{2}$ if the ADM mass of the Kerr-like wormholes is considered.
\end{abstract}

\footnotetext{
*Electronic address: amirctp12@gmail.com

${ }^{\dagger}$ Electronic address: kimet.jusufi@unite.edu.mk

${ }^{\ddagger}$ Electronic address: ayanbanerjeemath@gmail.com

$\S$ Electronic address: hansrajs@ukzn.ac.za
} 


\section{INTRODUCTION}

In the context of general relativity wormholes act as tunnel-like structures connecting two different regions in spacetime. This phenomenon necessitates solving Einstein's equations in a reversed manner to obtain solutions admitting wormholes. Studies on this topic can be divided into two classes: one relating to the Euclidean wormholes [1-4] and the other concerned with the Lorentzian ones. Historically, the notion of wormhole physics may be traced back to 1916 by Flamm [5], soon after the discovery of the Schwarzschild solution. Einstein and Rosen [6] proposed a mathematical construction in order to eliminate coordinate or curvature singularities, eventually introduced a bridge-like structure connecting two identical sheets known as the Einstein-Rosen bridges. This mathematical representation of physical space was not successful as a model for particles rather it emerged as the prototype wormhole in gravitational physics. Subsequently, Wheeler $[7,8]$ revived the subject in the 1950s where the Kruskal-Szekeres coordinates were employed to describe the geometry of the Schwarzschild wormhole, although these wormholes were at the quantum scale. Wheeler coined the term wormhole and later his solutions were transformed into Euclidean wormholes by Hawking [4] and others.

Interest in traversable Lorentzian wormholes has been on the incline recently following the stimulating work of Morris and Thorne [9]. In this proposal it is possible for observers to freely traverse wormholes and time travel is admissable. They initially introduced concepts as pedagogic tools for teaching general relativity where the Einstein field equations are solved by constructing the spacetime metric first and then deducing the stress-energy tensor components. In order to construct such traversable wormhole solutions demands the existence of exotic matter whose stress-energy tensor components violate the null, weak, and the strong energy conditions $[9,10]$, at least in a neighborhood of the wormhole throat. For a more detailed review the reader may consult $[11,12]$ and references therein. While the introduction of exotic matter may appear unpalatable such matter fields exist in the quantum theory of the gravitational field (namely the Casimir effect and Hawking evaporation [13]) as well as in scalar-tensor theories. In fact wormhole geometries violate the averaged energy conditions [11].

Historically various attempts have been made to avoid appealing to exotic matter but all were in vain within the context of general relativity. An advance in this discussion was 
to consider minimizing the violation of the energy conditions, namely, the volume integral quantifier $[14,15]$, which quantifies the total amount of matter violating the energy conditions. In this regard Nandi et al. [16] considered an exact integral quantifier for matter violating the averaged null energy condition (ANEC). Kuhfittig [17] reported that the region consisting of exotic matter can be made arbitrarily small. Therefore, it is not an easy task to search for promising candidates for exotic matter because all classical forms of matter are believed to obey the standard energy-conditions. Dark energy models with exotic equations of state have been proposed due to the formation and the evolution of the present structures in the Universe. While observational and experimental support is presently lacking, strong theoretical arguments for dark matter have been advanced principally to address the anomalous accelerated expansion of the universe problem. Consequently, much effort has been made to obtain stable wormhole solutions within these new paradigms. These include models supported by the phantom energy [18-20], the cosmological constant [21-23], generalized or modified Chaplygin gas [24-26]. However, in some alternative or modified theories of gravity, the requirement of exotic matter can be minimized or even completely avoided. For instance, it was shown that the matter threading the wormhole throat satisfies all of the energy conditions by Harko et al. [27], and it is the higher order curvature terms, interpreted as a gravitational fluid, that support the wormhole geometry. In [28], wormhole solutions have also been found with matter satisfying the null energy condition were nonminimal curvature-matter coupling in a generalized $f(R)$ modified theory of gravity. These kind of solutions were also found in various modified theories, e.g., Einstein-Gauss-Bonnet [29], Born-Infeld gravity [30], Einstein-Cartan [31], and trace of the energy-momentum tensor squared gravity [32].

The detection of gravitational waves (GWs) by the advanced Laser Interferometer Gravitational Wave Observatory (aLIGO) [33] and Advanced Virgo [34] detectors offers exciting opportunities towards the study of the nature of black holes. The detection of GWs from five binary black holes (BBHs) demonstrated that stellar-mass black holes really exist in our universe. However, the complete structure of the spacetime inside the light ring and near-horizon is still opaque from the current observations. Thus, the physics in strong gravitational field, e.g., near black holes is still an important topic not only in theoretical but also observational investigations. Analysing the physical nature of black holes requires the actual detection of the event horizon. To confirm the presence of event horizons a number 
of tests have been performed that help reveal exciting physics and astrophysics possibilities [35-37]. The evidence is compelling however not conclusive [38]. Another important phenomenological feature of black holes is their shadow. The concept of the rotating black hole shadow was suggested by Bardeen [39], with the idea of the optical image of black hole appearing due to the strong gravitational lensing effect. Black holes are expected to cast shadows on the bright background. Current observations suggest that most galaxies contains supermassive black holes at their centers $[40,41]$ and that galaxies are rotating. This means that the black hole at the center of a galaxy also possesses a spin and in this environment it is interesting to investigate the nature of the black hole by analyzing the shadow that they cast on the bright background. Motivated by present observational missions [42], the study of the black hole shadow has received significant attention. Various configurations of black hole solutions, such as the shadow of the Schwarzschild black hole [43, 44], have been investigated. The investigation of the shadow for several black holes in the context of modified theories of gravity and higher-dimensional theories have received attention in [46-66]. Shadow casting by the Kerr-like black holes in modified gravity theory has been addressed recently in [67]. Different approaches to investigate the black hole shadow has been addressed so far in the literature, e.g., photon regions based description of shadow [45], Hioki and Maeda approach to calculate the observables of shadow [48], new method to perform general-relativistic ray-tracing for shadow images [68], coordinate-independent method [69], in presence of plasma [70], analytic description of shadow [71].

The main motivation of the paper is to investigate the shadows of Kerr-like wormholes obtained by Bueno et al. [72], where the Kerr black hole turns out to be a wormhole solution that reproduces Kerr's spacetime away from the throat. The same procedure outlined was followed by Damour and Solodukhin to obtain the Schwarzschild-like wormhole [73]. The detection of ringdown frequencies from the black holes provides precise tests that astrophysical black holes are verily described by the Kerr spacetime. Therefore, we conduct an analysis of shadow for the Kerr-like wormholes. In addition, it is expected that the shadow of the Kerr-like wormholes can be used to probe the true nature of wormholes by Very Long Baseline Interferometry (VLBI) observations. In this direction a recent paper has been devoted to the study of the shadow boundary associated with the outer spherical orbits [74] and later this work was developed by [75]. Ellis wormholes have been analyzed by using the images of wormholes surrounded by optically thin dust in [76]. The shadow of rotating 
wormholes in a plasma environment has been studied in the literature by the authors [77]. Recently, the shadow of a class of charged wormholes solutions in EMDA theory has been studied in [78].

The paper is organized as follows: after a brief introduction in Sect. I, we review the Kerr-like wormhole solution and construct embedding diagrams to represent a wormhole in Sect. II. In Sect. III, we evaluate the geodesic equations and derive the photon trajectories around the wormhole in Sect. IV. Section V is devoted to constructing the shadow images of Kerr-like wormholes. A discussion on the results is included in Sect. VI.

\section{KERR-LIKE WORMHOLES AND EMBEDDING DIAGRAMS}

We commence with the Kerr-like wormhole spacetime which was obtained as a toy model by performing a modification on the Kerr metric similar to that of Damour and Solodukhin for a Schwarzschild black hole [73]. This spacetime class was introduced by Bueno et al. [72] and the metric in Boyer-Lindquist coordinates $(t, r, \theta, \phi)$ is given by

$$
\begin{aligned}
d s^{2} & =-\left(1-\frac{2 M r}{\Sigma}\right) d t^{2}-\frac{4 M a r \sin ^{2} \theta}{\Sigma} d t d \phi+\frac{\Sigma}{\hat{\Delta}} d r^{2} \\
& +\Sigma d \theta^{2}+\left(r^{2}+a^{2}+\frac{2 M a^{2} r \sin ^{2} \theta}{\Sigma}\right) \sin ^{2} \theta d \phi^{2}
\end{aligned}
$$

where $\Sigma$ and $\hat{\Delta}$ are expressed as follows

$$
\Sigma=r^{2}+a^{2} \cos ^{2} \theta, \quad \hat{\Delta}=r^{2}+a^{2}-2 M\left(1+\lambda^{2}\right) r .
$$

This spacetime contains a family of parameters where $a$ and $M$, respectively corresponds to the spin and the mass of the wormhole and $\lambda^{2}$ is the deviation parameter which accounts for the deviation from the Kerr spacetime. In other words, for any non vanishing $\lambda^{2}$ the new metric differs from the Kerr metric. The Kerr spacetime may be recovered when $\lambda^{2}=0$. The throat of the Kerr-like wormhole (2) can be evaluated by equating the $\hat{\Delta}$ to zero, which gives

$$
r_{+}=M\left(1+\lambda^{2}\right)+\sqrt{M^{2}\left(1+\lambda^{2}\right)^{2}-a^{2}} .
$$

This expression represents a special surface or region that connects two different asymptotically flat regions. Now we can construct the embedding diagrams to represent a Kerr-like wormhole and extract some useful information by considering an equatorial slice, $\theta=\pi / 2$ 
and a fixed moment of time, $t=$ a constant. The metric has the form

$$
d s^{2}=\frac{d r^{2}}{1-\frac{b(r)}{r}}+R^{2} d \phi^{2}
$$

where

$$
R^{2}=r^{2}+a^{2}+\frac{2 M a^{2}}{r}, \text { and } b(r)=2 M\left(1+\lambda^{2}\right)-\frac{a^{2}}{r} .
$$

We embed the metric (4) into three-dimensional Euclidean space to visualize this slice and the spacetime can be written in cylindrical coordinates as

$$
d s^{2}=d z^{2}+d R^{2}+R^{2} d \phi^{2}=\left[\left(\frac{d R}{d r}\right)^{2}+\left(\frac{d z}{d r}\right)^{2}\right] d r^{2}+R^{2} d \phi^{2}
$$

The combination of (4) and (6) generate the equation for the embedding surface which is given by

$$
\frac{d z}{d r}= \pm \sqrt{\frac{r}{r-b(r)}-\left(\frac{d R}{d r}\right)^{2}}
$$

Now, plugging the value of (5) into (7) the above relation assumes the form

$$
\frac{d z}{d r}= \pm \sqrt{\frac{M\left[2 r^{7} \lambda_{1}+2 a^{2} r^{3}\left(2 r^{2}+a^{2}\right)-4 M a^{2} \lambda_{1} r^{4}-M a^{4}\left(r^{2}+a^{2}\right)+2 M a^{4} \lambda_{1} r\right]}{r^{3}\left(r^{3}+a^{2} r+2 M a^{2}\right)\left(r^{2}+a^{2}-2 M \lambda_{1} r\right)}},
$$

where we have put $\lambda_{1}=1+\lambda^{2}$ for convenience. Note that the integration of (8) cannot be accomplished analytically. Invoking numerical techniques allows us to illustrate the wormhole shape given in Fig. 1. In the limit $a \rightarrow 0$, we obtain

$$
z= \pm \sqrt{8 M\left(1+\lambda^{2}\right)\left(r-2 M-2 M \lambda^{2}\right)}
$$

for the integral of (8). In addition, on setting the deviation parameter to zero, i.e., $\lambda^{2}=0$, the Schwarzschild solution

$$
z= \pm \sqrt{8 M(r-2 M)}
$$

is regained. The problem may be simplified by considering a slowly rotating Kerr-like wormhole solution. For this purpose we are interested in approximate solutions by considering a series expansion, say, around $a$ and $\lambda^{2}$ in Eq. (8). The solution can be approximated as follows

$$
z= \pm\left\{\sqrt{8 M(r-2 M)}-\frac{\lambda^{2} \sqrt{2 M}}{4 M^{3 / 2} r(r-2 M)^{3 / 2}}\left[\sqrt{2} \arctan \left(\frac{r-2 M}{\sqrt{M}}\right)(r-2 M)^{3 / 2} r a^{2}+\chi\right]\right.
$$



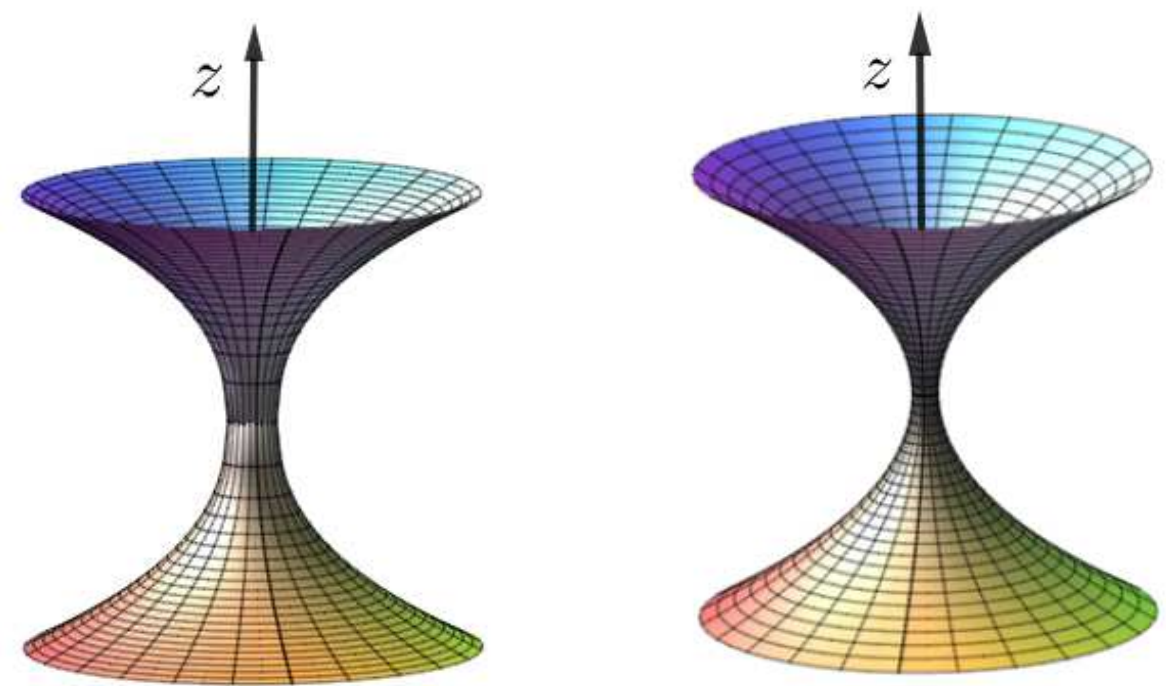

FIG. 1: Plot showing the embedding diagrams of the Kerr-like wormholes. (Left) Rotating Kerrlike wormhole: $z$ as a function of $r$ with $\phi$ direction for $M=1$ and $a=1$. (Right) Slowly rotating Kerr-like wormhole: plot of $z$ as a function of $\rho$ with $\phi$ direction for $M=1.2, a=0.01$ and $\lambda^{2}=0.01$.

$$
\left.+\frac{a^{2} \sqrt{2 M}}{16 r^{2} M^{3 / 2}(r-2 M)^{1 / 2}}\left[3 \sqrt{2} \arctan \left(\frac{r-2 M}{\sqrt{M}}\right)(r-2 M)^{1 / 2} r^{2}+\zeta\right]\right\},
$$

where

$$
\zeta=16 M^{5 / 2}-4 M^{3 / 2} r+6 r^{2} M^{1 / 2}
$$

and

$$
\chi=24 M^{5 / 2} r^{2}-32 M^{7 / 2} r+4 M^{5 / 2} a^{2}-4 M^{3 / 2} r^{3}-8 a^{2} M^{3 / 2} r+2 r^{2} a^{2} \sqrt{M} .
$$

Finally, let us introduce a new variable $\rho$, via

$$
r=2 M\left(1+\lambda^{2}\right)+\frac{\lambda^{2}-1}{2 M} a^{2}+\frac{\rho^{2}}{2 M} .
$$

The graphical representation of (14) with the $\phi$ direction can be seen from Fig. 1, which shows the visual image of the slowly rotating Kerr-like wormhole.

\section{A. ADM mass}

Now let us compute the ADM mass for the Kerr-like wormhole. We compute it for asymptotically flat spacetime and it will be similar to that of axisymmetric spacetime. We 
consider the asymptotic flat spacetime

$$
d s_{\Sigma}^{2}=\psi(r) d r^{2}+r^{2} \chi(r)\left(d \theta^{2}+\sin ^{2} \theta d \varphi^{2}\right)
$$

where we have identified

$$
\psi(r)=\frac{1}{1-\frac{2 M\left(1+\lambda^{2}\right)}{r}}, \quad \text { and } \quad \chi(r)=1 .
$$

In order to compute the ADM mass, we use the approach which used in [75],

$$
M_{A D M}=\lim _{r \rightarrow \infty} \frac{1}{2}\left[-r^{2} \chi^{\prime}+r(\psi-\chi)\right] .
$$

On substituting the values in (17) and after computing the limit we get the ADM mass for the wormhole,

$$
M_{A D M}=M\left(1+\lambda^{2}\right) .
$$

Note that this is the mass of the Kerr-like wormhole as seen by an observer located at the asymptotic spatial infinity. We will use this mass to explain the physical processes throughout the paper.

\section{GEODESICS IN KERR-LIKE WORMHOLES}

The spacetime of rotating Kerr-like wormholes is generally characterized by the four constants of motion, i.e., the Lagrangian $\mathcal{L}$, the energy $E$, the angular momentum $L_{z}$ and the Carter constant $\mathcal{K}$. The geodesic equations of a test particle having rest mass $m_{0}$ can be derived by using these conserved quantities and the Hamilton-Jacobi equation. We can derive the geodesic equations for $t$ and $\phi$ coordinates with the help of conserved quantities, $E=-p_{t}$ and $L_{z}=p_{\phi}$, which turns out

$$
\begin{aligned}
\Sigma \frac{d t}{d \sigma} & =-a\left(a E \sin ^{2} \theta-L_{z}\right)+\frac{\left(r^{2}+a^{2}\right) \mathcal{P}}{r^{2}+a^{2}-2 M r} \\
\Sigma \frac{d \phi}{d \sigma} & =-\left(a E-L_{z} \csc ^{2} \theta\right)+\frac{a \mathcal{P}}{r^{2}+a^{2}-2 M r}
\end{aligned}
$$

where $\mathcal{P}=\left(r^{2}+a^{2}\right) E-a L_{z}$. The other geodesic equations, i.e., for $r$ and $\theta$ coordinates can be computed by using the Hamilton-Jacobi method. Since the geodesics of the test particle in the background of Kerr-like wormholes spacetime (1) satisfy the Hamilton-Jacobi equation [79], which is given by

$$
\frac{\partial S}{\partial \sigma}=-\frac{1}{2} g^{\mu \nu} \frac{\partial S}{\partial x^{\mu}} \frac{\partial S}{\partial x^{\nu}},
$$


where $\sigma$ is the affine parameter and $S$ is the Jacobian action with the following separable ansatz:

$$
S=\frac{1}{2} m_{0}^{2} \sigma-E t+S_{r}(r)+S_{\theta}(\theta)+L_{z} \phi .
$$

Here $S_{r}$ and $S_{\theta}$ are functions of $r$ and $\theta$, respectively. On substituting Eq. (21) into Eq. (20) and after some straightforward computations we obtain the following forms of the geodesic equations

$$
\begin{aligned}
& \Sigma \frac{d r}{d \sigma}= \pm \sqrt{\mathcal{R}}, \\
& \Sigma \frac{d \theta}{d \sigma}= \pm \sqrt{\Theta},
\end{aligned}
$$

where the terms $\mathcal{R}$ and $\Theta$ in (22) are expressed as follows

$$
\begin{aligned}
\mathcal{R} & =\frac{\left[r^{2}+a^{2}-2 M\left(1+\lambda^{2}\right) r\right]\left\{\mathcal{P}^{2}-\left(r^{2}+a^{2}-2 M r\right)\left[\mathcal{K}+\left(L_{z}-a E\right)^{2}+m_{0}^{2} r^{2}\right]\right\}}{r^{2}+a^{2}-2 M r}, \\
\Theta & =\mathcal{K}+\cos ^{2} \theta\left(a^{2} E^{2}-L_{z}^{2} \csc ^{2} \theta-m_{0}^{2} a^{2}\right) .
\end{aligned}
$$

These geodesic equations determine the trajectories of a test particle in background of the Kerr-like wormholes spacetime. Physically, the quantity $E$ is the energy required for a distant observer to place the test particle in orbit around the Kerr-like wormhole.

\section{SPHERICAL PHOTON ORBITS AROUND KERR-LIKE WORMHOLES}

Consider the case of photons released from a bright source and which are moving towards the wormhole that is placed between the observer and the light source. The possible trajectories of the photons around the wormhole are: (i) falling into the wormholes, (ii) scattered away from the wormholes to infinity, and (iii) critical geodesics which separate the first two sets, also known as unstable spherical orbits. It turns out that in the observer's sky, the plunged photon geodesics form dark spots whereas the scattered photon geodesics form bright spots. The critical photon geodesic trajectories form a dark region in the observer's sky in the presence of a bright background which is referred to as the wormhole hole shadow.

Our principal objective is to evaluate these critical geodesics or unstable spherical orbits. In order to obtain the boundary of the wormhole shadow, it is necessary to work out the radial motion of photons around the wormholes. Here, we consider a photon as a massless test particle, therefore $m_{0}=0$. The radial geodesic equation can be rewritten as

$$
\left(\Sigma \frac{d r}{d \sigma}\right)^{2}+V_{e f f}=0
$$



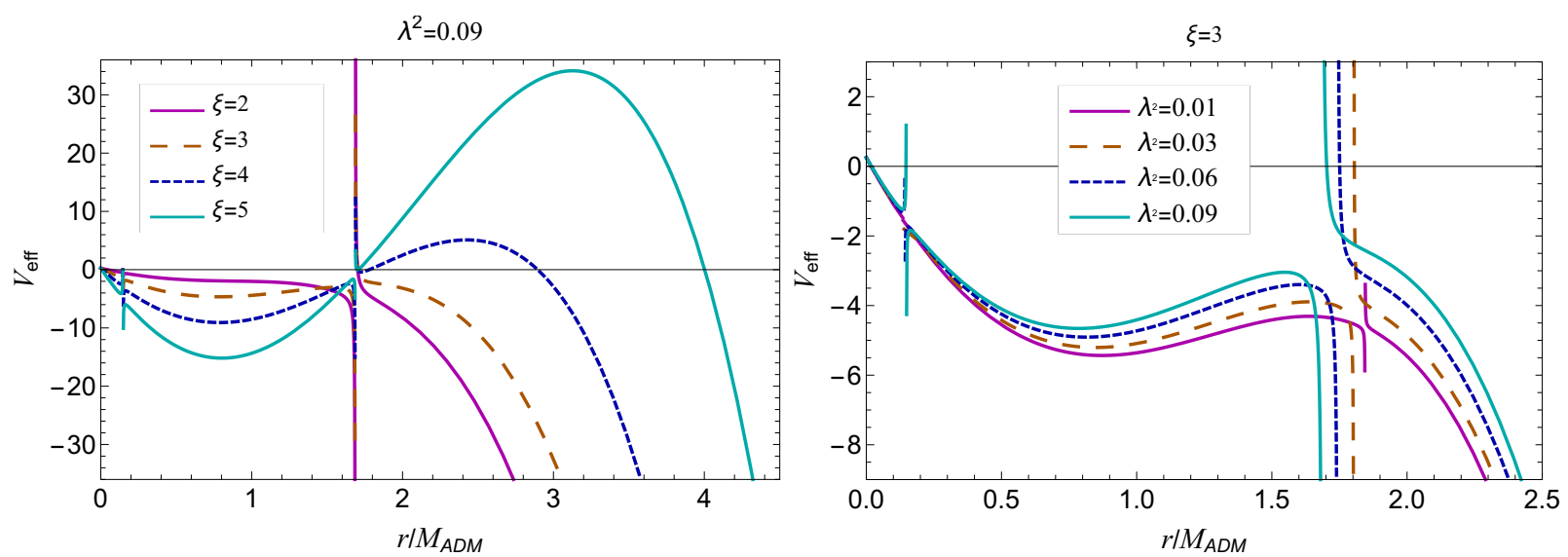

FIG. 2: Plot illustrating the nature of effective potential in Kerr-like wormholes by varying the impact parameter $\xi$ and the deviation parameter $\lambda^{2}$.

where $V_{\text {eff }}$ represents the effective potential that describes the trajectories of the photons around the wormhole. However, the different bounds of the effective potential determine the various trajectories depending on the magnitude of the angular momentum. Now we express the effective potential of the photons in terms of two independent impact parameters such that $\xi=L_{z} / E$ and $\eta=\mathcal{K} / E^{2}$. The effective potential can be written as follows

$$
\begin{aligned}
V_{e f f}= & -\frac{E^{2}\left[r^{2}+a^{2}-2 M\left(1+\lambda^{2}\right) r\right]}{r^{2}+a^{2}-2 M r}\left[\left(r^{2}+a^{2}-a \xi\right)^{2}\right. \\
& \left.-\left(r^{2}+a^{2}-2 M r\right)\left[\eta+(\xi-a)^{2}\right]\right] .
\end{aligned}
$$

It is easy to observe that the effective potential has a dependency of the deviation parameter $\lambda^{2}$ that affects the trajectories of photons. We plot effective potential with radius for the Kerr-like wormhole in Fig. 2 by varying the parameter $\xi$ as well as the deviation parameter $\lambda^{2}$. We use the ADM mass $\left(M_{A D M}\right)$ to plot the effective potential of the Kerr-like wormholes. We find that the effective potential has different potential barriers according to the variation of parameters which increase with parameter $\xi$ as well as with the deviation parameter $\lambda^{2}$. On the other hand, the boundary of the shadow can be determined by the unstable spherical photon orbits corresponding to the highest maximum value (supremum) of the effective potential $V_{\text {eff }}$. The following standard conditions can be used to determine the unstable spherical photon orbits

$$
V_{\text {eff }}=0, \quad V_{\text {eff }}^{\prime}=0,
$$

where prime (') represents derivatives with respect to $r$. In terms of $\mathcal{R}$, the unstable spherical 
photon orbits conditions transform as follows

$$
\mathcal{R}=0, \quad \mathcal{R}^{\prime}=0
$$

Now on using Eqs. (23) and (27), we immediately obtain the expressions of the impact parameters

$$
\begin{aligned}
\xi & =\frac{a^{2}(r+M)+r^{2}(r-3 M)}{a(M-r)}, \\
\eta & =\frac{4 M a^{2} r^{3}-r^{4}(r-3 M)^{2}}{a^{2}(M-r)^{2}}
\end{aligned}
$$

where $r$ is the radius of unstable spherical photon orbits. Interestingly, Eq. (28) determines the critical locus of the impact parameters which can be considered as the set of unstable photon orbits. As it can be seen from Eq. (28) that the impact parameters have a dependency on the spin $a$ and the mass $M$. When we compare our computed expressions of the impact parameters with the Kerr spacetime, we find them indistinguishable. However, there is a dependency of deviation parameter $\lambda^{2}$ on the impact parameters through the ADM mass by using the relation, $M=M_{A D M} /\left(1+\lambda^{2}\right)$. If we compute the impact parameters for $a=0$ (nonrotating case), we obtain the following relation

$$
\eta=27 M^{2}-\xi^{2} \quad \text { with } \quad r=3 M
$$

This result is similar to that of the Schwarzschild spacetime one. Although we are using the ADM mass which turns out that there is a dependency on deviation parameter $\lambda^{2}$. The apparent image or shadow of the wormhole can be obtained by looking for the unstable photon orbits which can be discussed more explicitly in the next section.

\section{SHADOW OF KERR-LIKE WORMHOLES}

In this section, our main goal is to construct and characterize the shadow of the Kerr-like wormholes. We turn our attention towards the observer's sky to detect the optical images cast by the Kerr-like wormholes. In preparation for the investigation of the shadow, consider a plane passing through the center of the wormhole and it's normal joining the center of the wormhole and the line of sight of an observer. We introduce new coordinates $(\alpha, \beta)$ [39], widely known as celestial coordinates that span a two-dimensional plane also known as celestial plane. Note that the coordinates $\alpha$ and $\beta$ corresponds to the apparent perpendicular 
distance of the image as seen from the axis of symmetry and the apparent perpendicular distance of the image from its projection on to the equatorial plane, respectively. With this construction, we obtain the projection of wormhole's throat on the observer's sky. The celestial coordinates for a distant observer can be written [39] in the form

$$
\begin{aligned}
& \alpha=\lim _{r \rightarrow \infty}\left(-r^{2} \sin \theta_{0} \frac{d \phi}{d r}\right), \\
& \beta=\lim _{r \rightarrow \infty}\left(r^{2} \frac{d \theta}{d r}\right),
\end{aligned}
$$

where $\theta_{0}$ represents the inclination angle between the rotation axis of the wormhole and the direction to the observer. By using Eqs. (19), (22), (23), and (30), the celestial coordinates transform into

$$
\begin{aligned}
& \alpha=-\xi \csc \theta_{0}, \\
& \beta= \pm \sqrt{\eta+a^{2} \cos ^{2} \theta_{0}-\xi^{2} \cot ^{2} \theta_{0}} .
\end{aligned}
$$

It is clear from Eq. (31) that the shapes of the Kerr-like wormhole's shadow depend not only on its spin $a$ but also on its inclination angle $\theta_{0}$. When the observer is located in the equatorial plane $\left(\theta_{0}=\pi / 2\right)$ of the wormhole, the expressions in Eq. (31) reduce to

$$
\begin{aligned}
& \alpha=-\xi, \\
& \beta= \pm \sqrt{\eta} .
\end{aligned}
$$

A dark region in the observer's sky can be obtained when the impact parameters $\eta$ and $\xi$ attains all the possible values. This dark region is known as the shadow of the wormhole. The nonrotating wormhole shadow can be constructed by the following expression

$$
\alpha^{2}+\beta^{2}=\frac{2 r^{2}\left(r^{2}-3 M^{2}\right)}{(M-r)^{2}},
$$

which implies that the shadow casts by a nonrotating wormhole $(a=0)$ has a circular shape with a radius $\sqrt{\frac{2 r^{2}\left(r^{2}-3 M^{2}\right)}{(M-r)^{2}}}$. In order to plot the shadows, we consider the ADM mass $\left(M_{A D M}\right)$ of the wormhole. The shadows of nonrotating wormhole can be seen from Fig. 3. The figure shows that the radius of shadow decreases with a small change in deviation parameter $\lambda^{2}$. Moreover, the radius of shadow is smaller than that of Schwarzschild black hole (cf. Fig. 3). In contrast for a rotating wormhole the sum of $\alpha^{2}+\beta^{2}$, takes the complicated form

$$
\alpha^{2}+\beta^{2}=\frac{a^{2}(M+r)^{2}+2 r^{2}\left(r^{2}-3 M^{2}\right)}{(M-r)^{2}} .
$$




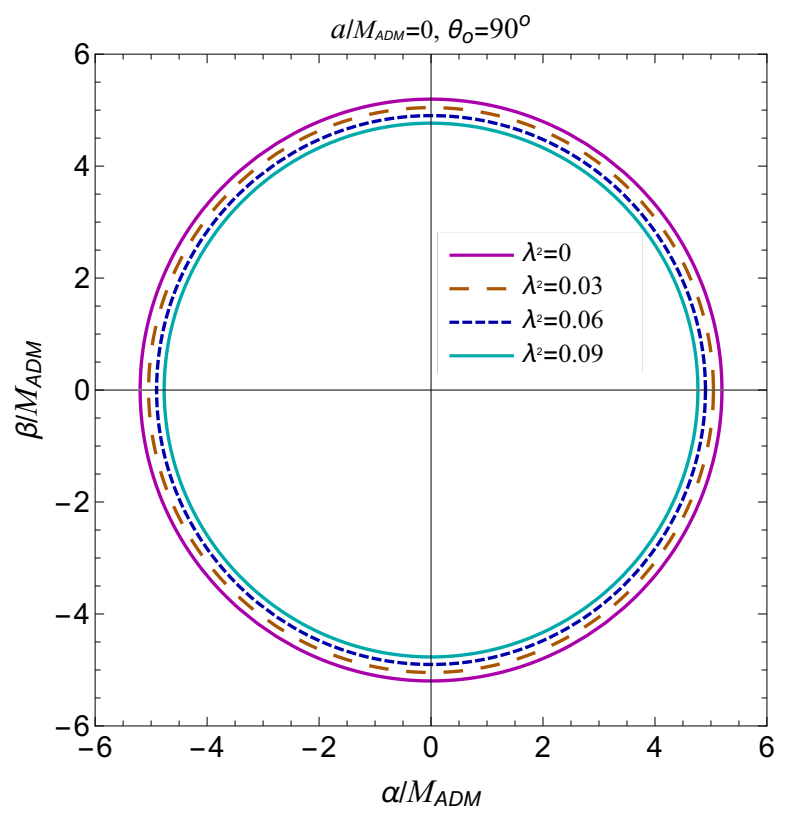

FIG. 3: Plot illustrating the shadow images of the nonrotating wormholes by varying the deviation parameter $\lambda^{2}$. Here $\lambda^{2}=0$ corresponds to the Schwarzschild black hole case $\left(M_{A D M}\right.$ is set equal to 1 for both of the spacetimes).

The presence of spin $a$ in the expression (34) results in manifestly different shapes of the shadow of the Kerr-like wormhole in comparison with the nonrotating one. It is noticeable that the expression (34) depends on deviation parameter $\lambda^{2}$ while the ADM mass of the wormhole is considered. This is because as we pointed out we shall refer to the ADM mass as the physical mass of the wormhole viewed by an observer located at spatial infinity. In order to see it more clearly, we construct shapes of the Kerr-like wormholes shadows for nonzero values of the spin $a$, the deviation parameter $\lambda^{2}$, and the inclination angle $\theta_{0}$. We plot the shadow images of the Kerr-like wormholes for different values of the spin $a$, the deviation parameter $\lambda^{2}$, and the inclination angle $\theta_{0}$ (cf. Figs. 4 and 5). Interestingly, we find that the shape of the shadow is oblate instead of being circular when compare with the nonrotating case. This oblateness or distortion in the shape arises because of the nonzero values of the spin $a$. In Fig. 4, we show the variation of the spin $a$ while the Fig. 5 depicts the effect of deviation parameter $\lambda^{2}$ on the shape of the shadow. It is clearly observed that the presence of the deviation parameter $\lambda^{2}$ influences the radius of the shadow.

An increase in the magnitude of spin $a$ increases the amount of distortion in the shadow of the Kerr-like wormhole (cf. Figs. 4 and 5). On the other hand when we fix the values 

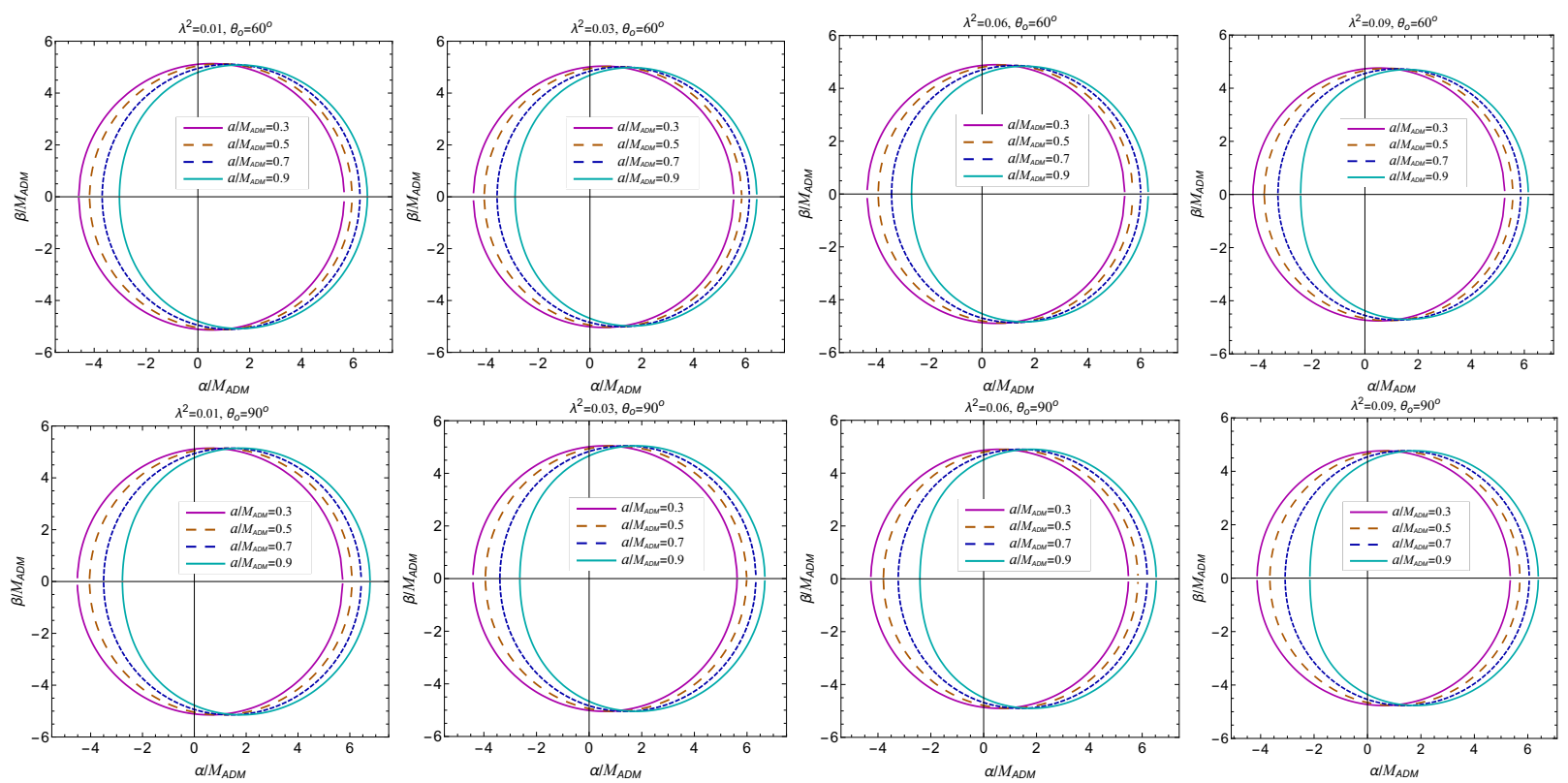

FIG. 4: Plot illustrating the shadow images of the Kerr-like wormholes by varying the spin $a$ for different values of deviation parameter $\lambda^{2}\left(M_{A D M}=1\right)$.
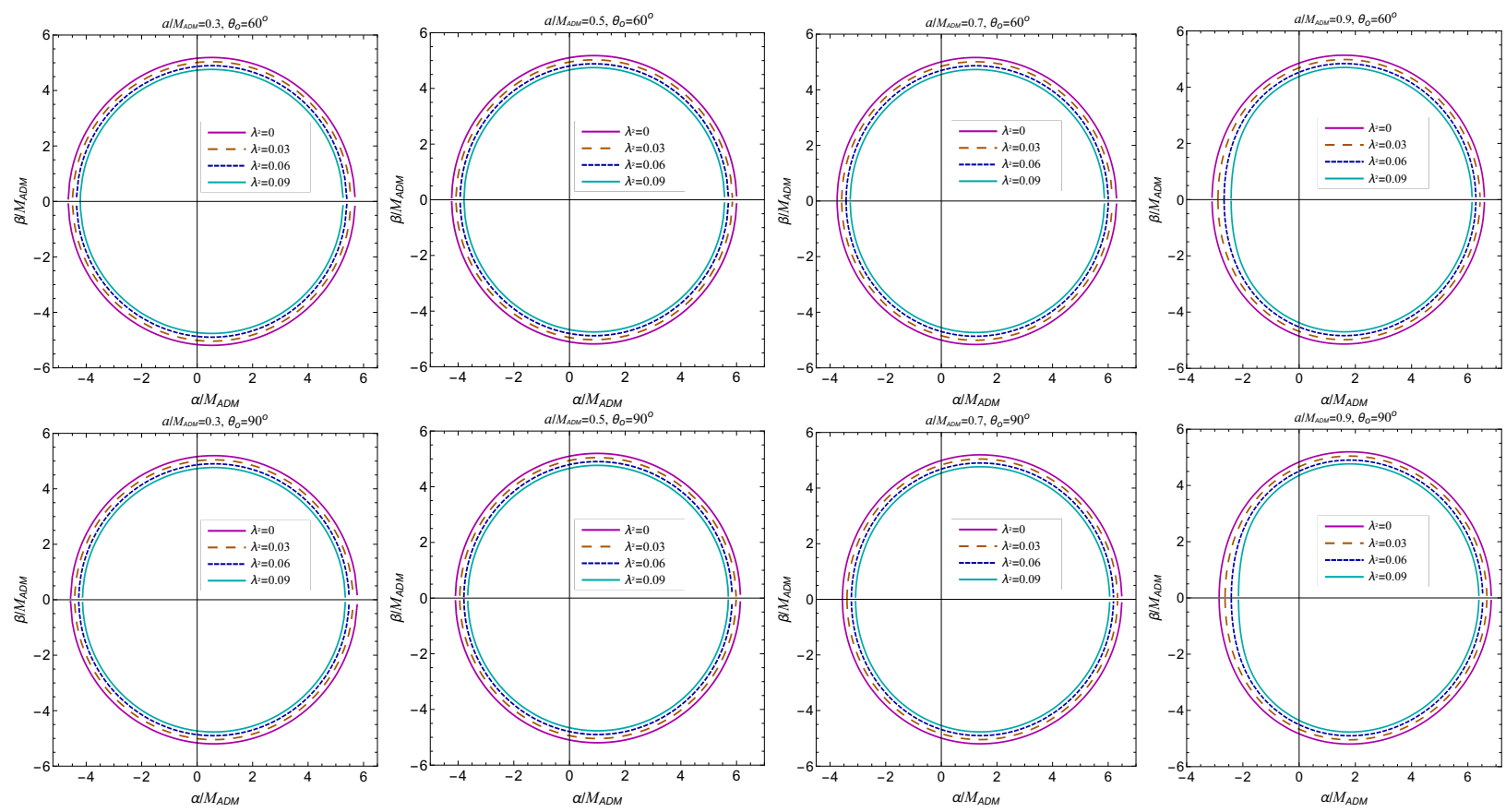

FIG. 5: Plot illustrating the shadow images of the Kerr-like wormholes by varying the deviation parameter $\lambda^{2}$. Here $\lambda^{2}=0$ corresponds to the Kerr black hole case $\left(M_{A D M}\right.$ is set equal to 1 for both of the spacetimes). 
of $a$ and at the same stage we do a change in $\lambda^{2}$ values that also increases the distortion in the shape. The shape is maximally distorted when the observer is located in equatorial plane as can be seen from Fig. 5. While we talk about the effect on the size or radius of the Kerr-like wormhole's shadow, we find that the radius is continuously decreasing with a small change in the magnitude of the deviation parameter $\lambda^{2}$ (cf. Fig. 5). We also include the case of the Kerr black hole $\left(\lambda^{2}=0\right)$ by considering the same ADM mass $\left(M_{A D M}=1\right)$ to see the difference in their shadow images (cf. Fig. 5). We discover that the shadow images of the Kerr-like wormholes are smaller in comparison to that of the Kerr black hole. In case of the Kerr spacetime, one can only observe the effect of spin $a$ on shadow, but in our case we have an additional parameter $\lambda^{2}$ which provides more images of the shadow so that extract more substantial information from them. Note that the actual size of the wormhole's shadow is bigger as compare to wormhole's geometrical size. Since the wormhole bends photon trajectories, therefore, the actual cross section of the shadow is greater than that of the geometrical one.

\section{CONCLUSION}

The discovery of quasar in 1960s provided compelling evidence for the existence of supermassive black holes located at least in some galactic binary systems in the centers of most of the large galaxies, e.g., the Milky Way and Messier 87 ostensibly have such objects, namely, Sgr A* and M87*. Supermassive black holes are considered as astrophysical black holes and they form from the gravitational collapse of matter. Besides, the detection of ringdown frequencies from the black holes provide precise tests that astrophysical black holes are accurately described by the Kerr spacetime. Motivated by these tests, we construct the theoretical analysis of the shadow for the Kerr-like wormholes. Therefore, observational investigation of the Kerr-like wormhole shadows will be very interesting and a useful tool to demonstrate the true nature of it. The observation of the shadow also provides a tentative way to determine the parameters governing the evolution of the wormholes. As it is well known one of the approaches to detect supermassive black holes is based on high resolution imaging of them. The Event Horizon Telescope which functions on the Very Long Baseline Interferometry technique, has been designed to resolve the problem of the detection of the supermassive black hole at the center of the Milky Way. This technique is able to achieve 
the angular resolution comparable to sub-millimeter wavelength diapason.

In this paper, we concentrate on the construction of the shadow cast by the Kerr-like wormholes. We have evaluated the test particle geodesics and determined the trajectories of photons around the Kerr-like wormhole. It has been noticed that the photons approaching the wormhole with sufficient angular momentum form unstable spherical orbits around it. In further investigation, we introduced the impact parameters that define the boundary of the shadow against the bright background. In order to visualize the optical images of the shadow of the Kerr-like wormhole, the celestial coordinates are evaluated. We have plotted these coordinates into the celestial plane to get the shadow images, eventually discussed the nonrotating and rotating cases of the wormhole's shadows. We have discovered that the radii of the nonrotating wormhole's shadows are smaller in comparison to that of the Schawrzschild black hole. We have obtained different shapes of the shadow for the Kerr-like wormholes by varying the parameters. We find that the shapes of the Kerr-like wormhole's shadow are oblate or distorted. Mainly, this distortion occurs due to the magnitude of the spin parameter $a$ and inclination angle $\theta_{0}$. The shapes are more distorted for the extreme value of the spin $a$ and when the observer is located into the equatorial plane. In addition, it is found that the radius of the shadow images is also sensitive to value of the deviation parameter $\lambda^{2}$; a small change in $\lambda^{2}$ decreases the radius of the shadow substantially. As a consequence the radius decreases with deviation parameter $\lambda^{2}$ in general. Our results provide the deviations when a comparison with the Kerr spacetime is taking on account by using the same ADM mass for both of the spacetimes. We have also discovered that the radii of the Kerr-like wormhole's shadows are smaller in comparison to that of the Kerr black hole. This important study will be helpful to extract the essential information about the Kerr-like wormhole and its existence as the astrophysical object. We are expecting that in upcoming years the direct images of wormholes will be observe by the Event Horizon Telescope.

\section{Acknowledgments}

M.A., A.B., and S.H. would like to thank University of KwaZulu-Natal and the National Research Foundation for financial support. We would like to thank the referees for useful 
comments and suggestions.

[1] S. Ruz, S. Debnath, A. K. Sanyal and B. Modak, Euclidean wormholes with minimally coupled scalar fields, Class. Quant. Grav. 30, 175013 (2013).

[2] N. Arkani-Hamed, J. Orgera and J. Polchinski, Euclidean wormholes in string theory, J. High Energy Phys. 0712, 018 (2007).

[3] S.W. Hawking, Quantum Coherence Down the Wormhole, Phys. Lett. B 195, 337 (1987).

[4] S.W. Hawking, Wormholes in Space-Time, Phys. Rev. D 37, 904 (1988).

[5] L. Flamm, Beitrage zur Einsteinschen Gravitationstheorie Phys. Z. 17, 448 (1916).

[6] A. Einstein and N. Rosen, The Particle Problem in the General Theory of Relativity, Phys. Rev. 48, 73 (1935).

[7] J. A. Wheeler, Geons, Phys. Rev. 97, 511 (1955).

[8] J. A. Wheeler, Geometrodynamics, (Academic Press, New York, 1962).

[9] M.S. Morris and K.S. Thorne, Wormholes in spacetime and their use for interstellar travel: A tool for teaching General Relativity, Am. J. Phys. 56, 395 (1988).

[10] M.S. Morris, K.S. Thorne and U. Yurtsever, Wormholes, Time Machines, and the Weak Energy Condition, Phys. Rev. Lett. 61, 1446 (1988).

[11] M. Visser, Lorentzian Wormholes: From Einstein to Hawking (American Institute of Physics, New York, 1995).

[12] F.S.N. Lobo, Exotic solutions in General Relativity: Traversable wormholes and 'warp drive' spacetimes, Classical and Quantum Gravity Research, 1-78, (2008).

[13] G. Klinkhammer, Averaged energy conditions for free scalar fields in flat spacetime, Phys. Rev. D 43, 2542 (1991).

[14] M. Visser, S. Kar and N. Dadhich, Traversable wormholes with arbitrarily small energy condition violations, Phys. Rev. Lett. 90, 201102 (2003).

[15] S. Kar, N. Dadhich and M. Visser, Quantifying energy condition violations in traversable wormholes, Pramana 63, 859 (2004).

[16] K. K. Nandi, Y. Z. Zhang and K. B. Vijaya Kumar, On volume integral theorem for exotic matter, Phys. Rev. D 70, 127503 (2004).

[17] P.K.F. Kuhfittig, Can a wormhole supported by only small amounts of exotic matter really be 
traversable?, Phys. Rev. D 68, 067502 (2003).

[18] F.S.N. Lobo, Phantom energy traversable wormholes, Phys. Rev. D 71, 084011 (2005).

[19] F.S.N. Lobo, Stability of phantom wormholes, Phys. Rev. D 71, 124022 (2005).

[20] O.B. Zaslavskii, Exactly solvable model of wormhole supported by phantom energy, Phys. Rev. D 72, 061303 (2005).

[21] F. Rahaman, M. Kalam, M. Sarker, A. Ghosh and B. Raychaudhuri, Wormhole with varying cosmological constant, Gen. Rel. Grav. 39, 145 (2007)

[22] J.P.S. Lemos, F.S.N. Lobo and S. Quinet de Oliveira, Morris-Thorne wormholes with a cosmological constant, Phys. Rev. D 68, 064004 (2003).

[23] M. Cataldo, S. del Campo, P. Minning and P. Salgado, Evolving Lorentzian wormholes supported by phantom matter and cosmological constant, Phys. Rev. D 79, 024005 (2009).

[24] S. Chakraborty and T. Bandyopadhyay, Modified Chaplygin traversable wormholes, Int. J. Mod. Phys. D 18, 463 (2009).

[25] M. Jamil, U. Farooq and M.A. Rashid, Wormholes supported by phantom-like modified Chaplygin gas, Eur. Phys. J. C 59, 907 (2009).

[26] P.K.F. Kuhfittig, A Single model of traversable wormholes supported by generalized phantom energy or Chaplygin gas, Gen. Rel. Grav. 41, 1485 (2009).

[27] T. Harko, F. S. N. Lobo, M. K. Mak and S. V. Sushkov, Modified-gravity wormholes without exotic matter, Phys. Rev. D 87, 067504 (2013).

[28] N. Montelongo Garcia and F. S. N. Lobo, Nonminimal curvature-matter coupled wormholes with matter satisfying the null energy condition, Class. Quant. Grav. 28, 085018 (2011).

[29] M. R. Mehdizadeh, M. Kord Zangeneh and F. S. N. Lobo, Einstein-Gauss-Bonnet traversable wormholes satisfying the weak energy condition, Phys. Rev. D 91, 084004 (2015).

[30] R. Shaikh, Wormholes with nonexotic matter in Born-Infeld gravity, Phys. Rev. D 98, 064033 (2018).

[31] M. R. Mehdizadeh and A. H. Ziaie, Einstein-Cartan wormhole solutions, Phys. Rev. D 95, 064049 (2017).

[32] P. H. R. S. Moraes and P. K. Sahoo, Nonexotic matter wormholes in a trace of the energymomentum tensor squared gravity, Phys. Rev. D 97, 024007 (2018).

[33] J. Aasi et al. [LIGO Scientific Collaboration], Advanced LIGO, Class. Quant. Grav. 32, 074001 (2015). 
[34] F. Acernese et al. [VIRGO Collaboration], Advanced Virgo: a second-generation interferometric gravitational wave detector, Class. Quant. Grav. 32, 024001 (2015).

[35] A.E. Broderick, A. Loeb and R. Narayan, The Event Horizon of Sagittarius A*, Astrophys. J. 701, 1357 (2009).

[36] A.E. Broderick, R. Narayan, J. Kormendy, E. S. Perlman, M.J. Rieke and S.S. Doeleman, The Event Horizon of M87, Astrophys. J. 805, no. 2, 179 (2015).

[37] R. Narayan and J. E. McClintock, Advection-Dominated Accretion and the Black Hole Event Horizon, New Astron. Rev. 51, 733 (2008).

[38] M.A. Abramowicz, W. Kluzniak and J.P. Lasota, No observational proof of the black hole event-horizon, Astron. Astrophys. 396, L31 (2002).

[39] J.M. Bardeen, Black holes, in Proceeding of the Les Houches Summer School, Session 215239, edited by C. De Witt and B.S. De Witt and B.S. De Witt (Gordon and Breach, New York, 1973).

[40] M.J. Rees, Black Hole Models for Active Galactic Nuclei, Ann. Rev. Astron. Astrophys. 22, $471(1984)$.

[41] J. Kormendy and D. Richstone, Inward Bound-The Search For Supermassive Black Holes In Galactic Nuclei, Ann. Rev. Astron. Astrophys. 33, 581 (1995).

[42] S. Doeleman, E. Agol, D. Backer et al., Imaging an event horizon: submm-VLBI of a super massive black hole, Astro2010: The Astronomy and Astrophysics Decadal Survey, Science White Papers, No. 68 (2009).

[43] J.L. Synge, The escape of photons from gravitationally intense stars, Mon. Not. R. Astron. Soc. 131, 463 (1966).

[44] J.P. Luminet Image of a spherical black hole with thin accretion disk, Astron. Astrophys. 75, $228(1979)$.

[45] A. Grenzebach, V. Perlick and C. Lammerzahl, Photon Regions and Shadows of KerrNewman-NUT Black Holes with a Cosmological Constant, Phys. Rev. D 89, 124004 (2014).

[46] R. Takahashi, Black hole shadows of charged spinning black holes, Publ. Astron. Soc. Jap. 57, $273(2005)$.

[47] C. Bambi and K. Freese, Apparent shape of super-spinning black holes, Phys. Rev. D 79, $043002(2009)$.

[48] K. Hioki and K.i. Maeda, Measurement of the Kerr Spin Parameter by Observation of a 
Compact Object's Shadow, Phys. Rev. D 80, 024042 (2009).

[49] S.W. Wei and Y.X. Liu, Observing the shadow of Einstein-Maxwell-Dilaton-Axion black hole, J. Cosmol. Astropart. Phys. 11 (2013) 063.

[50] C. Bambi and N. Yoshida, Shape and position of the shadow in the $\delta=2$ Tomimatsu-Sato space-time, Class. Quant. Grav. 27, 205006 (2010).

[51] L. Amarilla, E.F. Eiroa and G. Giribet, Null geodesics and shadow of a rotating black hole in extended Chern-Simons modified gravity, Phys. Rev. D 81, 124045 (2010).

[52] L. Amarilla and E. F. Eiroa, Shadow of a rotating braneworld black hole, Phys. Rev. D 85, 064019 (2012).

[53] L. Amarilla and E. F. Eiroa, Shadow of a Kaluza-Klein rotating dilaton black hole, Phys. Rev. D 87, 044057 (2013).

[54] A. Yumoto, D. Nitta, T. Chiba and N. Sugiyama, Shadows of Multi-Black Holes: Analytic Exploration," Phys. Rev. D 86, 103001 (2012).

[55] A. Abdujabbarov, F. Atamurotov, Y. Kucukakca, B. Ahmedov, and U. Camci, Shadow of Kerr-Taub-NUT black hole," Astrophys. Space Sci. 344, 429 (2013).

[56] F. Atamurotov, A. Abdujabbarov and B. Ahmedov, Shadow of rotating non-Kerr black hole, Phys. Rev. D 88, 064004 (2013).

[57] Z. Li and C. Bambi, Measuring the Kerr spin parameter of regular black holes from their shadow, J. Cosmol. Astropart. Phys. 01 (2014) 041.

[58] P.V.P. Cunha, C.A.R. Herdeiro, E. Radu, and H.F. Runarsson, Shadows of Kerr black holes with scalar hair, Phys. Rev. Lett. 115, 211102 (2015).

[59] T. Johannsen, Photon Rings around Kerr and Kerr-like Black Holes, Astrophys. J. 777, 170 (2013).

[60] A. Abdujabbarov, M. Amir, B. Ahmedov, and S.G. Ghosh, Shadow of rotating regular black holes, Phys. Rev. D 93, 104004 (2016).

[61] M. Amir and S.G. Ghosh, Shapes of rotating nonsingular black hole shadows, Phys. Rev. D 94, 024054 (2016).

[62] R. Kumar, B.P. Singh, M.S. Ali, and S.G. Ghosh, Rotating black hole shadow in Rastall theory, arXiv:1712.09793.

[63] B.P. Singh, Rotating charge black holes shadow in quintessence, arXiv:1711.02898.

[64] U. Papnoi, F. Atamurotov, S.G. Ghosh, and B. Ahmedov, Shadow of five-dimensional rotating 
Myers-Perry black hole, Phys. Rev. D 90, 024073 (2014).

[65] M. Amir, B. P. Singh and S. G. Ghosh, Shadows of rotating five-dimensional charged EMCS black holes, Eur. Phys. J. C 78, 399 (2018).

[66] B.P. Singh and S.G. Ghosh, Shadow of Schwarzschild-Tangherlini black holes, Annals Phys. 395, 127 (2018).

[67] H. M. Wang, Y. M. Xu and S. W. Wei, Shadows of Kerr-like black holes in a modified gravity theory, J. Cosmol. Astropart. Phys. 03 (2019) 046.

[68] Z. Younsi, A. Zhidenko, L. Rezzolla, R. Konoplya and Y. Mizuno, New method for shadow calculations: Application to parametrized axisymmetric black holes, Phys. Rev. D 94, 084025 (2016).

[69] A. A. Abdujabbarov, L. Rezzolla and B. J. Ahmedov, A coordinate-independent characterization of a black hole shadow, Mon. Not. Roy. Astron. Soc. 454, 2423 (2015).

[70] V. Perlick, O. Y. Tsupko and G. S. Bisnovatyi-Kogan, Influence of a plasma on the shadow of a spherically symmetric black hole, Phys. Rev. D 92, 104031 (2015).

[71] O. Y. Tsupko, Notes on analytical treatment of black hole shadow, Int. J. Mod. Phys. D 27, 1844020 (2018).

[72] P. Bueno, P.A. Cano, F. Goelen, T. Hertog and B. Vercnocke, Echoes of Kerr-like wormholes, Phys. Rev. D 97, 024040 (2018).

[73] T. Damour and S. N. Solodukhin, Wormholes as black hole foils, Phys. Rev. D 76, 024016 (2007).

[74] P.G. Nedkova, V.K. Tinchev, and S.S. Yazadjiev, Shadow of a rotating traversable wormhole, Phys. Rev. D 88, 124019 (2013).

[75] R. Shaikh, Shadows of rotating wormholes, Phys. Rev. D 98, 024044 (2018).

[76] T. Ohgami and N. Sakai, Wormhole shadows, Phys. Rev. D 91, 124020 (2015).

[77] A. Abdujabbarov, B. Juraev, B. Ahmedov and Z. Stuchlík, Shadow of rotating wormhole in plasma environment, Astrophys. Space Sci. 361, 226 (2016).

[78] M. Amir, A. Banerjee and S. D. Maharaj, Shadow of charged wormholes in Einstein-Maxwelldilaton theory, Annals Phys. 400, 198 (2019).

[79] S. Chandrasekhar, The Mathematical Theory of Black Holes (Oxford University Press, New York, 1992).

[80] K. Jusufi, A. Banerjee, G. Gyulchev and M. Amir, Eur. Phys. J. C 79, 28 (2019). 